\title{
Discussion
}

\section{Alzheimer Research Forum Live Discussion: Alzheimer's: A triple whammy. Why are so many neurodegenerative diseases single, double, or triple amyloidoses ${ }^{1}$}

\author{
http://www.alzforum.org/res/for/journal/trojanowski/default.asp
}

\begin{abstract}
John Q. Trojanowski and Mark P. Mattson led this live discussion on 22 October 2003 to discuss the major commonality among many neurodegenerative diseases.
\end{abstract}

Participants: Gabrielle Strobel, Moderator (Alzheimer Research Forum), Pete Nelson (University of Pennsylvania, Philadelphia), Davi Bock (University of Vermont, Burlington), Marcos Marques (University of Cincinnati, Ohio), Mikolaj Pawlak (Department of Neurology, University of Medical Science, Poznan, Poland), Alexei Koudinov (Russian Academy of Medical Sciences), Edward Zamrini (University of Alabama, Birmingham), Diego Forero (National University of Colombia), Mark Mattson (National Institute on Aging, Baltimore, Maryland), John Trojanowski (University of Pennsylvania, Philadelphia).

John Q. Trojanowski: Well, I will get things going by asking a question, which is: Do we underestimate the burden of misfolded proteins in neurodegenerative diseases by defining misfolding as equivalent to inclusions by relying too much on microscopy, since much of the abnormal protein accumulations may be in the form of oligomers that are not evident by microscopy, but could be detected biochemically?

Mark Mattson: One issue I am interested in is why dopaminergic neurons seem exquisitely sensitive to overload of the proteasome. The emerging findings suggest that simply increasing levels of wild-type synuclein is sufficient to cause Parkinson disease (PD). It seems as though the proteasome of dopaminergic neurons is sitting on the edge of a cliff with regard to the amount of ubiquitinated synuclein it can handle.

John Q. Trojanowski: In response to Mark, do we really know that dopaminergic neurons are so much more sensitive than other neurons to the accumulation of misfolded proteins?

Mark Mattson: We need some way of "titrating" abnormal protein accumulations and then monitoring the consequences with regard to various endpoints of interest - in my case, calcium regulation and oxidative stress.

Gabrielle Strobel: May I put a question out to everyone, especially John and Mark? How common are double and triple neurodegenerative brain amyloidoses? Do you have a sense of that?

John Q. Trojanowski: As we look in greater detail, especially with biochemistry allowing formic acid insolubility to be a surrogate for what is likely to be an amyloid fibril-containing protein pool, I think double and triple brain amyloidoses may be the rule rather than the exception. 
Gabrielle Strobel: Fascinating, John. So standard examination of pathological samples simply tended to overlook "other" amyloids? Certainly Kurt Jellinger (Institute of Clinical Neurobiology, Austria) has long pointed to overlapping pathologies among dementias, correct?

John Q. Trojanowski: Using more traditional morphological criteria, Alzheimer disease (AD) is the most common triple brain amyloidosis, since there is amyloid- $\beta(\mathrm{A} \beta)$ plaque amyloid and tau tangle amyloid in all cases, and over 50 percent also have $\beta$-synuclein Lewy body amyloid.

Alexei Koudinov: But until now, people rarely called tau changes in AD "amyloid”, right, John?

John Q. Trojanowski: Terminology has varied but there is consensus now, I think, that tangles, plaques, Lewy bodies, and other fibrillar deposits are all amyloids formed by different building-block proteins.

Alexei Koudinov: In biochemical/biophysical terms ( $\beta$-pleated secondary structure, etc.), does tau chemistry in $\mathrm{AD}$ fit the amyloidosis definition? Literature avoided such definitions in the past; am I right?

John Q. Trojanowski: If you look at recent work from Michel Goedert and Anthony Crowther (Medical Research Council, UK) in PNAS [1], you will find their paper with elegant data proving that tau fibrils are amyloids, and the same has been reported by many labs for $\beta$-synuclein fibrils.

Gabrielle Strobel: What could be the mechanisms underlying the convergence of more than one brain amyloid in many neurodegenerative diseases?

John Q. Trojanowski: In mutation-bearing people, it is a mutation, but in sporadic disease it is less clear what the causes of protein misfolding and amyloidosis are.

Mark Mattson: The mechanisms are likely multifactorial. For example, oxidative stress can promote abnormal folding of proteins and protein aggregation. Overload of the proteasome seems important, and, of course, the data from studies of disease-causing mutations have provided important clues - overproduction of the long form of $\mathrm{A} \eta$ in $\mathrm{AD}$, impaired ubiquitin-mediated proteolysis of synuclein in PD, etc. As aging is the major risk factor for the "double and triple amyloidoses", a focus should be on the age-related molecular changes.

John Q. Trojanowski: I agree with Mark on possible mechanisms in sporadic and possibly familial neurodegenerative diseases with brain amyloid deposition.

Gabrielle Strobel: Mark, you are interested in effects of diet on aging and neurodegeneration. Do you have data on links between dietary compounds and protein misfolding/aggregation?

Mark Mattson: Gabrielle, not yet. However, Tuck Finch has recently shown that $\mathrm{A} \beta$ deposition is decreased in $\mathrm{A} \beta \mathrm{PP}$ mutant mice on a reduced calorie diet. We have found that dietary restriction upregulates protein chaperones in neurons, which would be expected to enhance their ability to deal with damaged and abnormal proteins such as $\mathrm{A} \beta$, tau, and synuclein. We will be testing the latter hypothesis in the coming months/years.

Edward Zamrini: Do we know 1) if/how much any of the novel, in vivo amyloid markers detect intracellular vs. extracellular amyloid/fibrillar deposits, and 2) the relative strength of detection of one type vs. another?

Gabrielle Strobel: I also wonder if this is another way to look at oxidative stress. I am often puzzled because it seems so obviously to spur neurodegeneration, but specific in vivo links to, say, $\mathrm{AD}$ pathogenesis, are less clear to me.

John Q. Trojanowski: Oxidation could alter protein conformation, leading to misfolding, and if sufficient amounts of the misfolded protein accumulate, amyloidosis could ensue. Indeed, folks like Chris Dobson (University of Cambridge) might say, give me any protein and tell me how much you want converted into $\beta$ pleated sheet-containing fibrils, and I can deliver them to you under the right in-vitro conditions.

Marcos Marques: Mark, looking the other way around, how would protein misfolding, particularly amyloidosis, affect neuronal metabolism?

Mark Mattson: Marcos, we know that protein misfolding can affect neuronal metabolism, but the mechanisms are in most cases unclear. Prion proteins are good examples. Of course, patients with AD and PD have impaired cellular energy metabolism, but that this 
is the direct result of protein misfolding has certainly not been established.

John Q. Trojanowski: Misfolded proteins may acquire a toxic function and have deleterious consequences thereby, or by accumulating in cells, they could pull down other proteins that are taken out of action, like the misfolded protein, leading to several losses of function due to the "sidelining" of the proteins in "garbage heaps" inside cells.

Alexei Koudinov: Mark and Gabrielle, regarding the above point on oxidative stress, I think it is very important to look at proteins (that we call amyloid here today) as normal functional elements of brain chemistry. Similarly, oxidative stress may well serve to modulate synaptic function and plasticity. This was proposed in several papers [2]. If so, all elements should be considered as one complex mechanism that we should attempt to understand.

John Q. Trojanowski: Again, I concur with Mark that factors which upregulate chaperones could protect cells from the toxicity associated with accumulations of misfolded proteins, be this amyloidosis or some other form of toxicity.

Mikolaj Pawlak: Regarding the relation between diet and $\mathrm{AD}$, is it not so that we are observing end results of diet applied for years and that is why it is difficult to observe a relationship between diet and disease progression in real time?

Gabrielle Strobel: Mark and John, all, there is a paper in Science about C. elegans living six times their normal life span, and being highly active [3]. They have a few mutations, mostly in insulin-related signaling. Does this suggest anything as to which signaling pathways should be checked for changes in human aging and neurodegeneration (although I am not aware of links between aging pathways and protein aggregation)?

John Q. Trojanowski: I am not certain which of the signaling pathways in the worms would be worth pursuing in human neurodegenerative diseases, but that is a good thought to consider.

Alexei Koudinov: Gabrielle, there is another recent paper (not in Science) which shows that a particular profile of lipoproteins is associated with extreme longevity. This may well be of importance in terms of a role for lipids and cholesterol in AD and lipoprotein signaling [4].

Mark Mattson: Gabrielle, mutations in the insulin signaling pathway increase life span and stress resistance in C. elegans. Apparently, the mutations relieve suppression of a forkhead transcription factor, resulting in increased expression of antioxidant enzymes and perhaps proteins involved in preventing protein damage and/or removing damaged/misfolded proteins.

Gabrielle Strobel: Fascinating, Mark. Gene expression studies of aging humans are coming along, and consistently seem to show differences (downregulation) of genes involved in stress response, DNA repair, etc. . . All this calls for more work on chaperones. As far as I know, chaperone genes tend not to come up much in screens for genes involved in neurodegeneration in, say, Drosophila or worms. How could one better study their role in these multiple amyloidoses?

Pete Nelson: One question seems to be: Which are chickens and which are eggs?

Diego Forero: Pete Nelson's question is very important. What is the primary mechanism in the amyloidosis? It may be only the visible consequences of other underlying pathogenic pathways.

John Q. Trojanowski: As to the chicken and egg question, I think things may go in either direction, since if one is born with a mutation in tau, this determines subsequent formation of tau amyloid, so you consider tau amyloid the egg because it can come first, i.e., disease begins at conception. But in sporadic disease, there may be many chickens laying eggs that break, damaging tau and precipitating aggregation to form tangles. Sorry to overdo the chicken/egg analogy, but that was how the question was framed.

Mark Mattson: Understanding the normal functions of synuclein, tau, and $\mathrm{A} \beta$ is important. It is of considerable interest, in this regard, that synuclein, tau, and $\mathrm{A} \beta \mathrm{PP}$ are axonal proteins. Their normal functions in axons and presynaptic terminals may provide important clues as to the earliest events in disease pathogenesis, as well as to how the abnormal protein aggregates arise.

Gabrielle Strobel: Does this point to axonal transport, then, as one possible unifying theme of what goes awry? 
Mark Mattson: Yes, axonal transport and synaptic vesicle recycling might be adversely affected early on in the course of the disease process.

John Q. Trojanowski: This is a good point on the possibility that in all of these diseases, there could be a disruption of transport because the disease proteins bind or perturb motor proteins, disassemble microtubules, or physically block traffic when large amyloid accumulations formed by tau or $\beta$-synuclein develop in axons as dystrophic neurites or Lewy neurites.

Diego Forero: The topics pointed out by Dr. Koudinov are also very important. We may try to understand the possible pathogenic pathways in amyloidosis, taking into account that these proteins are key regulators of neural function, not only "bad" amyloids.

Alexei Koudinov: To Diego and Pete on primary factor: I believe that I attempted to address this question through the prism of functional role for "amyloid" proteins in normal synaptic function/plasticity. For example, with regard to oxidative stress and nor$\mathrm{mal} /$ pathological amyloid biochemistry, an interesting sequence of events is proposed by A. Kontush, which is that $\mathrm{A} \beta$ initially serves as an antioxidant, but its increased production as antioxidant leads to the peptideaggregated pro-oxidative form.

Marcos Marques: Did anybody look at amyloid animal models for changes in parameters like diet and exercise as a means to reverse the neuronal metabolism imbalance, and at the molecular level, is it possible to determine, then, how amyloid affects neuronal metabolism?

Gabrielle Strobel: John, as an esteemed pathologist, what do you think of Larry Goldstein's (University of California, San Diego) argument that many of the swellings one commonly sees in neurodegenerative diseases are axonal blockages that induce, not accompany, damage?

John Q. Trojanowski: Mark, what has become of "synaptosis"? This was the concept that the disease protein may lead to programmed death of processes, and I think you and others proposed this possibility a while ago.

Gabrielle Strobel: John, do you mean a local apoptosis program in dendrites and terminals?
John Q. Trojanowski: Gabrielle, as you may recall from my recent commentary on the papers from the Brady and Goldstein laboratories [5,6], I was pleased to see the concept of axonal transport-induced degeneration extended from $\mathrm{AD}$, where it has been around for over 10 years, to other disorders such as polyQ diseases, and I think it plausible that impairments in axonal transport could be drivers for degeneration in multiple aging-related neurodegenerative disorders.

Mark Mattson: The evidence that activation of apoptotic cascades occurs in synapses, axons, and dendrites is quite strong, and it is clear that these cascades can propagate to the cell body, culminating in death. It is also becoming evident that apoptotic cascades can have local effects on synaptic function and structural remodeling in the absence of cell death. The possible links between the amyloid proteins under consideration here and apoptotic processes remain to be determined, although we do know that $\mathrm{A} \beta$ can induce "synaptic apoptosis", at least in cell culture and synaptosome preparations.

Diego Forero: Here is a reference about the concept of synaptic apoptosis developed by Mattson et al., 1998 [7].

John Q. Trojanowski: One would assume a progression from oligomers, to protofibrils, to fully formed amyloid fibrils based on in vitro studies, and this process could unfold anywhere in a cell. Indeed, we have shown by stereology [8] that only five percent of the area occupied by abnormal tau immunoreactivity in AD is in tangles, while $95 \%$ is in the dystrophic processes, which means that it is surprising that tangles correlate with dementia, because most of the tau pathology is in processes which are not normally counted in correlative studies.

Gabrielle Strobel: Charlie Glabe (University of California, Irvine) reported an antibody that appears to recognize mid-size oligomers of a number of fibrillogenic proteins. Would that be a useful tool to study the synergy you propose between different aggregation-prone proteins? Does the antibody stain in vivo sections of different disease brains?

Alexei Koudinov: I was recently reviewing literature on a functional role of $\mathrm{A} \beta / \mathrm{A} \beta \mathrm{PP}$ (with regard to a unifying role for cholesterol in synaptic degeneration), and found Askansas et al., 1992 [9] and Torroja et al., 
1999 [10]. Do the above imply that we miss a more general point while talking about a neurodegeneration commonality - a role for proteins in synaptic machinery?

Gabrielle Strobel: John and Mark, does your thinking say anything about where along the way of aggregation the damage happens to the synapse? Oligomers? Protofibrils? Fibrils? Do you consider this question important at all?

Alexei Koudinov: Gabrielle, we come to the conclusion that amyloid fibrils can damage synaptic plasticity and that diffuse amyloid has no such effect. For me, the major question is what causes the change in $\mathrm{A} \beta$ biology. When we understand this, we will be able to reverse amyloid by affecting the primary cause.

Diego Forero: Some recent references about the possible neuroprotective roles of amyloid proteins $[11,12]$.

John Q. Trojanowski: And this "synaptic apoptotic" damage could be compounded by impairments in axonal transport caused by an accumulation of insoluble tau or $\beta$-synuclein, because transport of key synaptic proteins would not occur normally, nor would trophic factors picked up at terminals be transported back to the perikaryon to sustain the viability of affected neurons.

Gabrielle Strobel: What is the role of ubiquitinproteasome degradation in this? It would seem to be a candidate for a shared mechanism, as it pops up in some way in all these diseases. But I see no overarching theme yet. Have I missed it? One interesting lead might be that Mike Ehlers (Duke University) studies proteasome degradation in dendrites as a mechanism involved in activity-dependent turnover of postsynaptic proteins.

Mark Mattson: Ubiquitin-mediated protein degradation seems to be at the heart of the problem in PD, as parkin is an E3 ligase and synuclein a substrate. In the case of $\mathrm{AD}, \mathrm{A} \beta$, and tau, the importance of the proteasome is less clear.

John Q. Trojanowski: Amyloidosis is a product of several mechanisms, including the oversupply of substrates for amyloidogenesis and the diminished clearance of substrates allowing accumulations that precipitate into amyloid fibrils under the appropriate in vitro conditions.
Davi Bock: John, regarding distribution of abnormal tau: Perhaps the tangles are more inflammatory than the dystrophic processes. One oft-neglected clue is that $\mathrm{A} \beta \mathrm{PP}$ has an iron response element [13] and an IL1 element [14] in its 5' UTR. I wonder if a unifying feature of these amyloidogenic proteins is that they are upregulated by inflammatory processes, and so when they themselves instigate an inflammatory process (due to stochastic misfolding which increases with age, for example), a positive feedback loop is initiated?

John Q. Trojanowski: In response to your query, oxidate/nitrative stress could be another chicken/egg phenomenon, in that it may increase with the accumulation of misfolded proteins, or if increased for other reasons, it could contribute to amyloidogenesis.

Alexei Koudinov: Diego's reference reminded me of another major observation: that PHF-like tau change occurs normally during the short ontogenic period of intense (and membrane cholesterol-demanding) nerve growth [15]. Can we call this condition amyloidosis? Nature developed it to serve neural/synaptic function. In the disease, therefore, this machinery may serve to compensate synaptic failure. Can it be called a pathological event? Or compensation would be a better definition. This will be available soon as a peer-reviewed publication.

Mark Mattson: The developmental changes in tau involve hyperphosphorylation and not aggregation of tau into filaments. It is clear that hyperphosphorylation of tau, per se, is not harmful to neurons, as it occurs normally in development. This is important, as it tends to dissociate phosphorylation from the pathogenic process in $\mathrm{AD}$ and related tauopathies.

Alexei Koudinov: Mark, there is certainly a borderline between natural normal compensation and a diseaselocked condition. Therefore, one should better and strictly define new amyloid proteins, so there will be no confusion.

John Q. Trojanowski: Mark, hyperphosphorylation or any phosphorylation of tau is not needed for in vitro tau amyloid fibril formation, and the tau gene mutations argue that abnormal tau phosphorylation is not the most upstream event in familial tauopathies. However, since the increasing phosphorylation decreases microtubule binding, the excess phosphorylation of tau would disengage it from microtubules and this will destabilize 
microtubules to impair axonal transport, while leading to pools of unbound tau that could reach concentration thresholds which result in tau fibrillization.

Mikolaj Pawlak: If you cannot stop amyloidogenesis, can you at least slow it down by limiting substrates? If yes, then how?

John Q. Trojanowski: Yes, and that is the purpose of $\beta$-secretase inhibitor therapies that are intended to reduce the levels of $\mathrm{A} \beta$ peptides, which are the substrates for amyloid fibril formation. Alternatively, vaccine therapies, and gene therapy to increase expression of neprylisin, would be other avenues of therapeutic intervention to reduce $\mathrm{A} \beta$ plaques in $\mathrm{AD}$.

Mikolaj Pawlak: I see. How about physiological processes influencing this pathway?

Gabrielle Strobel: John and Mark, can you tell us more about these aggregation-busting compounds you mention in the article?

Alexei Koudinov: John, with regard to $\mathrm{A} \beta$-lowering therapies, one should be sure that no normal pathway is affected"a subject deserving study.

Gabrielle Strobel: The new drug Velcade is a proteasome inhibitor and now in trials for prostate cancer. I wonder what would happen if it got into the brain of people with breaks in the blood-brain barrier (BBB)?

Mark Mattson: Yes, if the Velcade gets into the brain, it would likely be bad for neurons. Of course, if you have life-threatening cancer, you might not be too worried about increased risk of neurodegenerative disorders.

Gabrielle Strobel: Mark, I totally agree. Indolent prostate cancer, though, is a different beast from active multiple myeloma...

Diego Forero: Two interesting articles about proteasome dysfunction and $\mathrm{AD}[16,17]$.

Gabrielle Strobel: Just to rock the boat at the end of the hour: I find the notion of a shared mechanism the least convincing in AD because of the spatial separation between tangles and plaques. Do you think that $\mathrm{A} \beta$ misfolding and fibrillization begins intraneuronally, where perhaps its aggregation could interact with tau and $\beta$-synuclein?
Pete Nelson: My own two cents, vis-à-vis Gabrielle's observation: Neuritic plaques seem to me to represent an important nidus of pathology, as (extracellular) amyloid plaques are directly apposed to (intracellular) neurofibrillary pathology, and degenerating neurites. Somehow, one is very directly interacting with the other.

Gabrielle Strobel: Yes, but Pete, how can they interact "very directly" when there is a cell membrane in the middle?

Pete Nelson: The cell membranes are compromised in neuritic plaques, as shown early on by Bob Terry (University of California, San Diego), Henryk Wisniewski (Institute for Basic Research, New York), and other ultrastructural microscopists.

John Q. Trojanowski: I would emphasize that what we see in the way of amyloid deposits through a microscope is the tip of the iceberg, in my view, since I expect that the $\mathrm{AD}$ brain is awash in variable levels of $\mathrm{A} \beta$, tau, and often $\beta$-synuclein oligomers, so interactions could well take place outside the field of view of a microscope.

Gabrielle Strobel: Can I ask another question about drug discovery based on your hypothesis, John and Mark? Does it open new avenues to make stronger drugs than vitamin $\mathrm{E}$ out of the oxidative damage knowledge?

Mark Mattson: Antioxidants continue to hold therapeutic potential and many laboratories are working to identify novel antioxidants which easily enter the brain and scavenge radicals. It is hard to say whether they will have a major impact on the disease process, although they are likely to have some benefit with few side effects.

Gabrielle Strobel: John, I am beginning to see this majority view now, that there is this sea of different aggregating species, few of which are visible with a microscope.

Alexei Koudinov: I agree with John about the iceberg tip, and look forward to more great data by scientists in many related fields.

Gabrielle Strobel: So, Mark, should we go on calorie restriction in the meantime? 
Mark Mattson: Gabrielle, yes, it is very likely that each of our brains would benefit from smaller or less frequent meals. In addition to helping your neurons deal with damaged proteins, dietary restriction upregulates expression of neurotrophic factors, particularly BDNF. Our data suggest that BDNF mediates several beneficial effects of dietary restriction in the brain, including neuroprotection and stimulation of neurogenesis.

Gabrielle Strobel: Let me thank you all for coming and for this fascinating discussion. We clearly need to revisit this topic.

\section{References}

[1] J. Berriman, L.C. Serpell, K.A. Oberg, A.L. Fink, M. Goedert and R.A. Crowther, Tau filaments from human brain and from in vitro assembly of recombinant protein show cross-beta structure, Proc. Natl. Acad. Sci. USA 100 (2003), 9034-9038.

[2] A. Kamsler and M. Segal, Hydrogen peroxide modulation of synaptic plasticity, J. Neurosci. 23 (2003), 269-276.

[3] N. Arantes-Oliveira, J.R. Berman and C. Kenyon, Healthy animals with extreme longevity, Science 302 (2003), 611. Alzforum news story: http://www.alzforum.org/new/detail.asp? id $=895$.

[4] J. Herz and D.K. Strickland, LRP: a multifunctional scavenger and signaling receptor, J. Clin. Invest. 108 (2001), 779-784.

[5] G. Szebenyi, G. Morfini, A. Babcock, M. Gould, K. Selkoe, D.L. Stenoien, M. Young, P.W. Faber, M.E. MacDonald, M.J. McPhaul and S.T. Brady, Neuropathogenic forms of huntingtin and androgen receptor inhibit fast axonal transport, Neuron $\mathbf{4 0}$ (2003), 41-52.

[6] S. Gunawardena, L.-S. Her, R.G. Brusch, R.A. Laymon, I.R. Niesman, B. Gordesky-Gold, L. Sintaath, N.M. Bonini and L.S.B. Goldstein, Disruption of axonal transport by loss of huntingtin or expression of pathogenic polyQ proteins in Drosophila, Neuron 40 (2003), 25-40.

[7] M.P. Mattson, J. Partin and J.G. Begley, Amyloid beta-peptide induces apoptosis-related events in synapses and dendrites,
Brain Res. 807 (1998), 167-176.

[8] T.W. Mitchell, J. Nissanov, L.Y. Han, E.J. Mufson, J.A. Schneider, E.J. Cochran, D.A. Bennett, V.M. Lee, J.Q. Trojanowski and S.E. Arnold, Novel method to quantify neuropil threads in brains from elders with or without cognitive impairment, J. Histochem. Cytochem. 48 (2000), 1627-1638.

[9] V. Askanas, W.K. Engel and R.B. Alvarez, Strong immunoreactivity of beta-amyloid precursor protein, including the betaamyloid protein sequence, at human neuromuscular junctions, Neurosci. Lett. 143 (1992), 96-100.

[10] L. Torroja, M. Packard, M. Gorczyca, K. White and V. Budnik, The Drosophila beta-amyloid precursor protein homolog promotes synapse differentiation at the neuromuscular junction, J. Neurosci. 19 (1999), 7793-7803.

[11] L.D. Plant, J.P. Boyle, I.F. Smith, C. Peers and H.A. Pearson, The production of amyloid beta peptide is a critical requirement for the viability of central neurons, J. Neurosci. $\mathbf{2 3}$ (2003), 5531-5535.

[12] T. Arendt, J. Stieler, A.M. Strijkstra, R.A. Hut, J. Rüdiger, E.A. Van der Zee, T. Harkany, M. Holzer and W. Härtig, Reversible paired helical filament-like phosphorylation of tau is an adaptive process associated with neuronal plasticity in hibernating animals, J. Neurosci. 23 (2003), 6972-6981.

[13] J.T. Rogers, J.D. Randall, C.M. Cahill, P.S. Eder, X. Huang, H. Gunshin, L. Leiter, J. McPhee, S.S. Sarang, T. Utsuki, N. Greig, D.K. Lahiri, R.E. Tanzi, A.I. Bush, T. Giordano and S.R. Gullans, An iron-responsive element type II in the 5' untranslated region of the Alzheimer's amyloid precursor protein transcript, J. Biol. Chem. 277 (2002), 45518-45528.

[14] J.T. Rogers, L.M. Leiter, J. McPhee, C.M. Cahill, S.S. Zhan, H. Potter and L.N. Nilsson, Translation of the Alzheimer amyloid precursor protein mRNA is up-regulated by interleukin-1 through 5'-untranslated region sequences, J. Biol. Chem. 274 (1999), 6421-6431.

[15] A.R. Koudinov and N.V. Koudinova, Cholesterol, synaptic function and Alzheimer's disease, Pharmacopsychiatry 36(Suppl 2) (2003), S107-S112.

[16] A.D. Hope, R. de Silva, D.F. Fischer, E.M. Hol, F.W. van Leeuwen and A.J. Lees, Alzheimer's associated variant ubiquitin causes inhibition of the $26 \mathrm{~S}$ proteasome and chaperone expression, J. Neurochem. 86 (2003), 394-404.

[17] S. Keck, R. Nitsch, T. Grune and O. Ullrich, Proteasome inhibition by paired helical filament-tau in brains of patients with Alzheimer's disease, J. Neurochem. 85 (2003), 115-122. 